\title{
Aspectos patológicos da retinopatia diabética
}

\author{
Pathological features of diabetic retinopathy
}

\author{
Zélia Maria da Silva Corrêa \\ Ralph Eagle Jr²
}

Descritores: Diabetes mellitus, Retinopatia diabética/fisiopatologia; Retinopatia diabética/ patologia; Neovascularização retiniana

\section{INTRODUÇÃO}

$\mathrm{O}$ diabetes mellitus continua sendo uma importante causa de cegueira e limitação visual no mundo atual ${ }^{(1-3)}$. A retinopatia diabética é a causa mais importante de cegueira em americanos entre 20 e 74 anos de idade ${ }^{(4)}$. Fatores ambientais importantes para o desenvolvimento da retinopatia diabética foram identificados ${ }^{(5-6)}$ apesar de um número crescente de evidências sugerirem um componente genético na retinopatia diabética ${ }^{(6)}$. Os avanços realizados nas últimas décadas com o objetivo de melhorar o entendimento da retinopatia diabética estimularam esta revisão sobre as alterações oculares do diabetes mellitus. Os dados para este artigo foram coletados em livros textos e a partir dos endereços eletrônicos: www.pubmed.org e www.scielo.br usando os seguintes descritores: "diabetic retinopathy" ou "retinopatia diabética", "pathology" ou "patologia" e "pathologic mechanisms" ou "fisiopatologia" sendo que o cruzamento destes descritores resultou em 112 citações que foram analisadas para esse artigo.

\section{Fisiopatologia do diabetes mellitus}

O diabetes mellitus tipo I, também chamado diabete juvenil ou insulinodependente, geralmente desenvolve-se de forma repentina antes dos 20 anos de idade. Esta doença é desencadeada pela destruição das células beta, secretoras de insulina nas ilhotas de Langerhans do pâncreas. Esta destruição é provavelmente de natureza auto-imune, mediada por linfócitos $\mathrm{T}$ citotóxicos ${ }^{(3,7)}$. Os níveis de insulina plasmática destes pacientes costumam ser baixos e os mesmos não são obesos. Esta doença é controlada com uso diário de insulina injetável e dieta.

Já o diabetes mellitus tipo II geralmente aparece de forma insidiosa após os 30 anos em pacientes obesos. Os níveis de insulina plasmática destes pacientes costumam ser normais ou até elevados. Apesar das ilhotas de Langerhans estarem intactas, a secreção de insulina fica comprometida 
assim como a capacidade de absorção periférica deste hormônio. Esta doença parece ser causada por um defeito no transporte das moléculas de glicose no citoplasma das células das ilhotas. Os receptores de glicose também estão reduzidos em número. A maioria dos pacientes com diabetes mellitus tipo II são bem controlados com hipoglicemiantes orais e $\operatorname{dieta}^{(6)}$. Estes pacientes, freqüentemente obesos, costumam melhorar com a perda de peso.

A retinopatia diabética é a complicação ocular mais importante de qualquer das formas de diabetes mellitus ${ }^{(8)}$. Quando são comparados grupos de diabéticos com doença tipo I e II de duração semelhante, a prevalência de retinopatia é mais alta no grupo de diabéticos tipo $\mathrm{I}^{(5,9)}$. Os pacientes com diabetes juvenil apresentam também uma maior chance de desenvolver doença proliferativa provavelmente porque esses costumam ter hiperglicemia mais grave. No Ambulatório de Endocrinologia Pediátrica da Santa Casa de São Paulo, a prevalência da retinopatia diabética em um grupo de diabéticos juvenis estudado, foi $17,3 \%{ }^{(10)}$. No geral, entretanto, uma porção significativa dos diabéticos que evoluem para a cegueira é do tipo II porque este tipo de diabetes é mais comum. A prevalência da retinopatia diabética em qualquer um dos grupos está relacionada com a duração da doença ${ }^{(10)}$. Cerca de $50 \%$ dos pacientes diabéticos insulino-dependentes por 15 anos ou mais apresentam retinopatia ${ }^{(5,7,9,11)}$.

Acredita-se que as mais importantes complicações do diabetes mellitus como: retinopatia, nefropatia e neuropatia, sejam devidas à hiperglicemia ${ }^{(5,8-9,12)}$. As evidências acerca do papel da hiperglicemia incluem estudos retrospectivos que correlacionam o desenvolvimento e progressão da retinopatia diabética com o mau controle glicêmico, evidenciado com a dosagem da hemoglobina glicosilada e dados clínicos como os publicados pelo "Diabetes Control and Complications Trial" (DCCT). Este estudo clínico randomizado mostrou que pacientes submetidos ao controle glicêmico rigoroso tiveram uma progressão menor da retinopatia ${ }^{(13-14)}$.

\section{Fisiopatologia da retinopatia diabética}

Os estágios progressivos da retinopatia diabética podem ser reconhecidos clinicamente. O estágio inicial conhecido como retinopatia de fundo, é caracterizado por: edema retiniano, microaneurismas capilares, hemorragias e exsudatos. A próxima fase é a pré-proliferativa, caracterizada por exsudatos algodonosos ou áreas de infarto retiniano com isquemia progressiva. A fase proliferativa é caracterizada por neovascularização da retina, disco óptico e íris ${ }^{(5,8)}$. Essa neovascularização desencadeia complicações como hemorragia vítrea e descolamento tracional da retina que levam à cegueira ${ }^{(2-3)}$.

A retinopatia diabética é a manifestação retiniana de uma microangiopatia sistêmica generalizada que pode ser observada na forma de edema de retina, exsudatos e hemorragias. Hemorragias intra-retinianas apresentam-se em formatos variados e são características desta fase $\mathrm{e}^{(3)}$. $\mathrm{O}$ formato e o aspecto clínico da hemorragia são determinados pela localização do extravazamento sangüíneo. Hemorragias em formato escafói- de ou de meia-lua costumam ser observadas em pacientes com formas mais avançadas de retinopatia. Hemorragias em chamade-vela ou em formato de farpa ocorrem quando o sangue se deposita no trajeto dos axônios da camada de fibras nervosas que se curvam em arcos superior e inferiormente à fóvea, paralelos à membrana limitante interna da retina $(\mathrm{MLI})^{(7)}$. Hemorragias de aspecto arredondado e borrado, estão localizadas nas camadas mais profundas da retina onde os eritrócitos ficam represados por axônios cuja orientação é perpendicular à MLI e à membrana de $\mathrm{Bruch}^{(15)}$. Apesar de algumas hemorragias em meia-lua refletirem um descolamento hemorrágico da MLI, a maioria das hemorragias em formato escafóide nos pacientes diabéticos são pré-retinianas ou sub-hialóideas. $\mathrm{O}$ sangue é colecionado entre a MLI e a face posterior do vítreo descolado, freqüentemente revestido por neovascularização. Hemorragias maciças que se misturam ao vítreo costumam ocorrer nos estágios mais avançados da doença e são uma importante causa de baixa visual ${ }^{(3,7,15)}$.

O edema retiniano e os exsudatos encontrados na retinopatia diabética de fundo refletem a quebra da barreira hematoretiniana. Estudos de fluorofotometria do vítreo mostraram que a quebra da barreira hemato-retiniana é uma das lesões funcionais mais precoces em olhos diabéticos ${ }^{(16)}$. Essa barreira protege os delicados tecidos neurais (fotorreceptores), da mesma forma que a barreira hemato-encefálica, da sobrecarga osmótica de um ambiente com excesso de líquido ${ }^{(3,16)}$. As aderências juncionais das células endoteliais dos vasos retinianos constituem a parte interna ou intra-retiniana dessa barreira o que impede o extravazamento de fluoresceína dos vasos retinianos saudáveis. $\mathrm{O}$ epitélio pigmentar da retina forma a parte externa dessa barreira pela firme aderência de guirlanda de barras terminais no ápice de suas células. Os coriocapilares que nutrem o terço avascular externo da retina são fenestrados e vazam fluoresceína profusamente. Devido a esta característica, o epitélio pigmentar da retina serve como

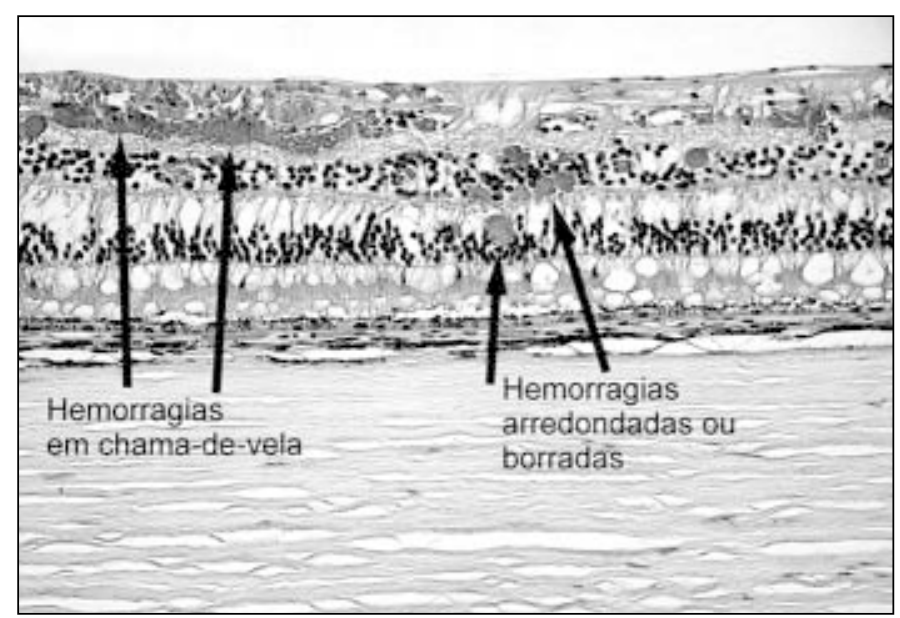

Figura 1 - Corte histológico da retina, coróide e esclera mostrando as alterações iniciais relacionadas à retinopatia diabética (10X). Note como as hemorragias apresentam diferentes formatos de acordo com a sua profundidade na retina 


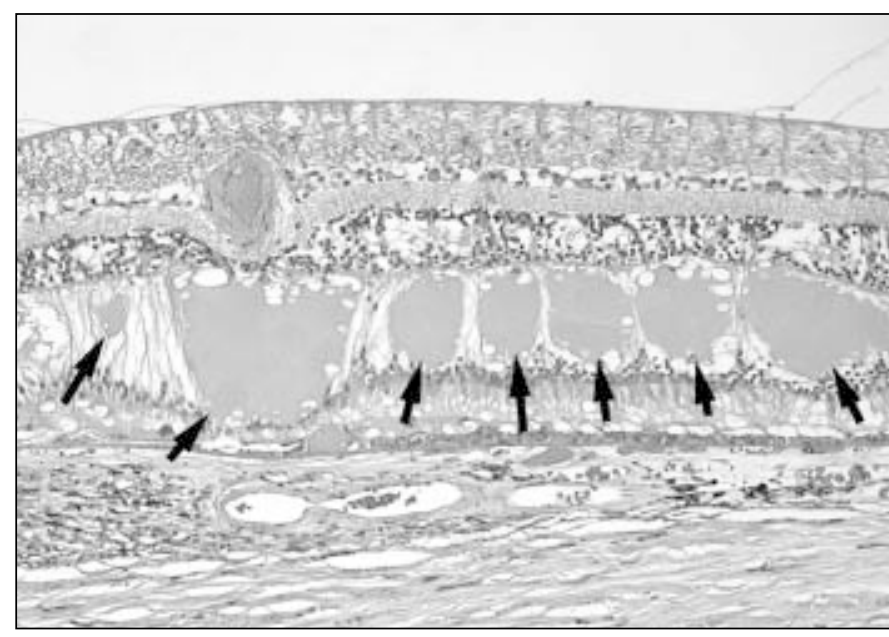

Figura 2 - Corte histológico da retina (10X) mostrando extensa atrofia da retina e depósitos de material denso, composto de lipídeos e proteínas (flechas) que correspondem clinicamente a exsudatos duros

barreira e proteção para a retina externa desse influxo de líquido. A incompetência da barreira hemato-retiniana permite o acesso de fluido rico em lipídeos e proteína ao parênquima retiniano causando edema e exsudação ${ }^{(17)}$. Na histopatologia, os exsudatos duros, amarelados e com aspecto de cera que caracterizam o diabetes mellitus se apresentam como coleções eosinofílicas de fluido protéico (vide flechas). Os exsudatos costumam se formar na camada plexiforme externa, uma região susceptível entre as duas fontes de suprimento sangüíneo da retina $^{(7,17)}$.

Exsudatos moles ou algodonosos tornam-se proeminentes na fase proliferativa da retinopatia diabética e servem como um marcador clínico de isquemia retiniana. Essas manchas algodonosas não são exsudatos verdadeiros mas sim áreas focais onde o fluxo axoplásmico normal da camada de fibras nervosas está bloqueado. Acredita-se que esse bloqueio focal do fluxo axoplásmico seja uma resposta à isquemia retiniana e provavelmente é causada pela trombose das arteríolas pré-capilares ${ }^{(17)}$. Os exsudatos algodonosos também ocorrem em outras doenças como: obstrução isquêmica da veia central da retina, retinopatia hipertensiva grave e, de forma isolada, em pacientes com Aids e doenças do colágeno como lúpus eritematoso sistêmico ${ }^{(7,15,17)}$. $\mathrm{Na}$ histopatologia, os exsudatos algodonosos são caracterizados pelo edema focal da camada de fibras nervosas na presença de corpos cistóides que são segmentos de células ganglionares infladas por axoplasma estagnado. Organelas intracelulares danificadas que se agregam no axoplasma formam uma estrutura nucleóide que simula um núcleo celular mas é eosinofílico ao invés de basofílico e são chamadas corpúsculos cistóides ${ }^{(15)}$.

Anormalidades características da retinopatia diabética podem ser demonstradas com uma técnica especializada de histopatologia chamada digestão retiniana com tripsina descrita inicialmente por Cogan e Kuwabara ${ }^{(18)}$. A enzima tripsina é usada para digerir as células da retina e os vasos sangüíneos são poupados devido a sua membrana basal que não é digerível. Os vasos sangüíneos são montados numa preparação plana e podem ser examinados diretamente após coloração com PAS (ácido periódico Schiff) que cora membrana basal ${ }^{(7,18)}$. Os capilares retinianos normais são compostos por células endoteliais que revestem o capilar e células murais ou pericitos localizados na cápsula da membrana basal perivascular. Os pericitos são células contráteis que regulam o calibre vascular e o fluxo da microcirculação retiniana. Normalmente existe uma célula endotelial para cada pericito no leito capilar da retina humana ${ }^{(18)}$. Pericitos são perdidos especialmente nas fases iniciais da retinopatia diabética ${ }^{(7,19)}$.

$\mathrm{O}$ verdadeiro mecanismo da perda dos pericitos permanece incerto. Alguns autores acreditam que a causa básica dessa perda seja o acúmulo intra-ocular de sorbitol produzido pela enzima aldose redutase na transformação de polióis. De acordo com esta hipótese, o sorbitol é formado quando níveis altos de glicose superam a capacidade metabólica o trajeto metabólico normal da glicólise. O sorbitol é incapaz de atravessar as membranas celulares e fica preso dentro da célula. Inibidores da aldose redutase parecem prevenir o desenvolvimento de retinopatia em modelos experimentais. O papel do sorbitol na morte dos pericitos tem sido discutido por aqueles que acreditam que a hiperglicemia tem um efeito inibitório na proliferação dessas células.

Preparações de retinas diabéticas com tripsina também revelam microaneurismas capilares, que são áreas totalmente acelulares no leito capilar associadas a um espessamento da membrana basal do capilar, evidente com o aumento da coloração com o PAS ${ }^{(15,20)}$. A essas áreas acelulares dos capilares correspondem áreas de não-perfusão, evidentes à angiografia fluoresceínica.

$\mathrm{O}$ interesse acerca dos eventos patológicos iniciais que levam à oclusão vascular tem aumentado ${ }^{(21)}$. Pesquisas em humanos e modelos experimentais mostram que é comum ocorrer uma estase de leucócitos nas fases iniciais dessa vasculopatia $^{(22)}$. Esse aumento do número de leucócitos coincide com o aumento da expressão de uma molécula de adesão intercelular retiniana (ICAM-1) e de CD 18 que promovem adesividade das células do sangue. Tal ocorrência sugere que um processo inflamatório pode estar associado às alterações sangüíneas e da parede vascular contribuindo para o processo de vaso-obliteração no diabetes ${ }^{(19,21-22)}$. Cortes histológicos seriados de áreas de não-perfusão capilar mostram afinamento isquêmico da retina que inclui todas as camadas nutridas pela artéria central da retina e a porção interna da camada nuclear interna ${ }^{(7,15)}$.

Microaneurismas retinianos aparecem como gomos de uva ou dilatações fusiformes nos capilares retinianos. Não se sabe exatamente como estes microaneurismas se formam. Vários microaneurismas parecem bem celulares sugerindo que a proliferação endotelial nos capilares possa estar envolvida com sua formação. Outro fator contribuinte na formação dos microaneurismas parece ser o enfraquecimento focal da parede vascular pela perda dos pericitos ${ }^{(3,7,15)}$. 


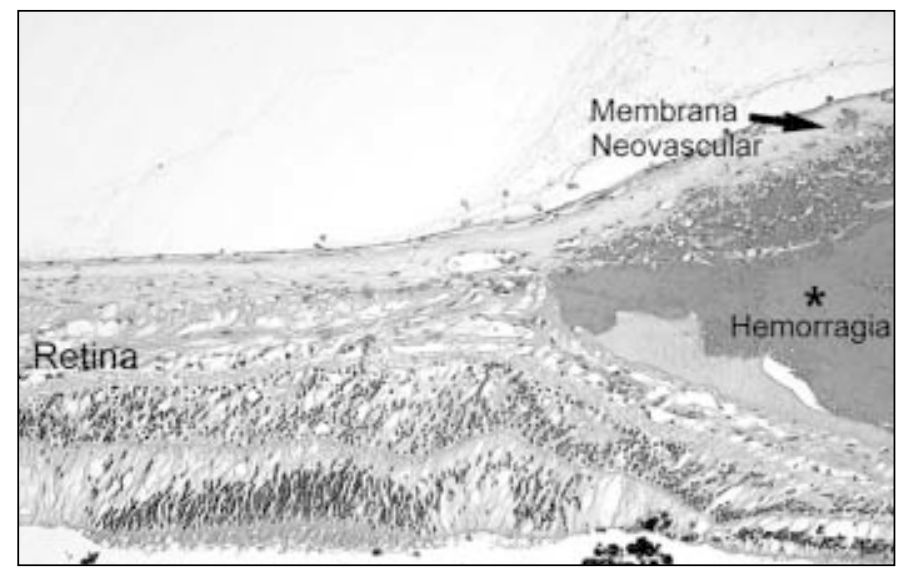

Figura 3 - Corte histológico da retina (10X) mostrando extensa atrofia e formação de uma camada fibro-vascular que causa tração e represa conteúdo hemorrágico

A perda dos pericitos dos capilares retinianos parece ter várias consequiências importantes. A falta das propriedades contráteis dos pericitos desregula os capilares retinianos acarretando alterações no fluxo sanguíneo. Além disso, estudos com cultura de tecidos mostraram que os pericitos têm um efeito inibidor na proliferação das células endoteliais, que é mediado pela transformação do fator de crescimento $\beta$. Esses experimentos sugerem que a perda dos pericitos leva à proliferação das células endoteliais e neovascularização pela perda do seu efeito inibitório ${ }^{(13)}$.

A retinopatia diabética proliferativa é marcada pela presença de proliferação neovascular que é um fator importante na patogênese de várias complicações que levam à cegueira, como o descolamento tracional da retina e hemorragia vítrea $^{(20)}$. Os novos vasos sanguíneos crescem dentro e sobre a retina e na face recentemente exposta pelo descolamento do vítreo posterior ${ }^{(7)}$. O estímulo para a neovascularização vítreoretiniana é sabidamente devido à liberação de um fator angiogênico produzido pela retina isquêmica. Estudos recentes revelaram que um fator angiogênico extremamente importante é o VEGF ("vascular endothelial growth factor" ou fator de crescimento vascular endotelial) $)^{(20-21)}$.

A síntese do VEGF é regulada pelos níveis de oxigênio no microambiente celular. O estímulo da síntese do VEGF pela hipóxia faz desta substância um possível fator responsável pela retinopatia proliferativa. O VEGF tem sido encontrado no fluido intra-ocular de pacientes com neovascularização ativa da retina e segmento anterior associado a várias doenças oculares associadas à isquemia retiniana. As concentrações de VEGF no vítreo e humor aquoso de pacientes com neovascularização ativa são mais altas que o necessário para estimular a proliferação endotelial "in vitro" e "in vivo"(21). Ao contrário do que acontece com indivíduos com neovascularização quiescente onde as concentrações do VEGF são muito baixas ou indetectáveis, a panfotocoagulação com "laser" bem sucedida produz uma queda significativa das concentrações do $\operatorname{VEGF}^{(19)}$.
Outros fatores de crescimento como fator básico de crescimento de fibroblastos (bFGF) podem ter um papel na neovascularização retiniana. Apesar de não ser sintetizado diretamente pela retina, este fator está presente no citoplasma das células retinianas e no tecido retiniano onde se liga à matriz extracelular. A lesão celular induzida pela isquemia prolongada pode levar à liberação desse fator ${ }^{(21)}$.

Esses fatores angiogênicos exercem seu efeito estimulatório nas células que compõem o lado venoso do leito capilar retiniano pré-existente. Com a proliferação das células estimuladas, ocorre a produção de colagenases e outras enzimas digestivas que dissolvem a membrana basal que envolve os capilares. Essas células migram, escorregando pelos espaços na parede dos vasos, em direção ao estímulo angiogênico. A neovascularização se inicia na retina onde é clinicamente evidenciada pela presença das IRMAs (anormalidades microvasculares intra-retinianas). Os novos vasos invadem a MLI e crescem sobre a superfície interna da retina. Apesar de a neovascularização ser incapaz de invadir o vítreo, ela pode crescer na face posterior do vítreo descolado ${ }^{(7,15,20)}$.

A neovascularização a partir do nervo óptico estende-se tipicamente até as paredes do espaço cônico conhecido como área de Martegiani que forma a parte posterior do Canal de Cloquet. Neovascularização vítreorretiniana estimula fibroplasia e fibrose vítrea que progride com o aumento do descolamento do vítreo posterior pela contração das membranas vítreas rasgando os delicados vasos sangüíneos. $\mathrm{O}$ resultado disso são hemorragias pré-retinianas e vítreas cuja organização produz ainda mais fibrose e tração. Considerando que o vítreo geralmente permanece aderente às grandes arcadas vasculares temporais, um descolamento localizado da mácula ocorre a partir da contração dessa ponte fibrovascular e tração resultante desta. Tração ântero-posterior também contribui para descolamento de retina ${ }^{(15,20)}$.

Olhos com retinopatia diabética proliferativa também são susceptíveis a neovascularização de íris e glaucoma neovascular $^{(23)}$. Estudos realizados sobre a concentração do VEGF no vítreo e aquoso de olhos de pacientes diabéticos revelaram que os níveis desse fator estavam sempre mais altos no vítreo. Tal fato explica porque a neovascularização de íris se inicia a partir da borda pupilar e ângulo onde a difusão do VEGF a partir do vítreo seria maior. Os neovasos proliferam sobre a superfície normal e avascular da íris aplanando e achatando sua arquitetura normal. Ocorre a formação de aderências permanentes entre a periferia da íris e o trabeculado, chamadas sinéquias anteriores periféricas. Miofibroblastos se proliferam, acompanhando os neovasos e consequentemente fornecendo os motivos para o fechamento angular e o desenvolvimento do ectrópio do esfíncter e do epitélio pigmentar da íris ${ }^{(7,23)}$. Alguns pacientes com diabetes apresentam uma forma mais branda de iridopatia, chamada vacuolização rendilhada do epitélio pigmentar da íris ${ }^{(24)}$. Essa vacuolização rendilhada é causada pelo acúmulo de glicogênio nos espaços cistóides do epitélio pigmentar da íris e ocorre somente em 


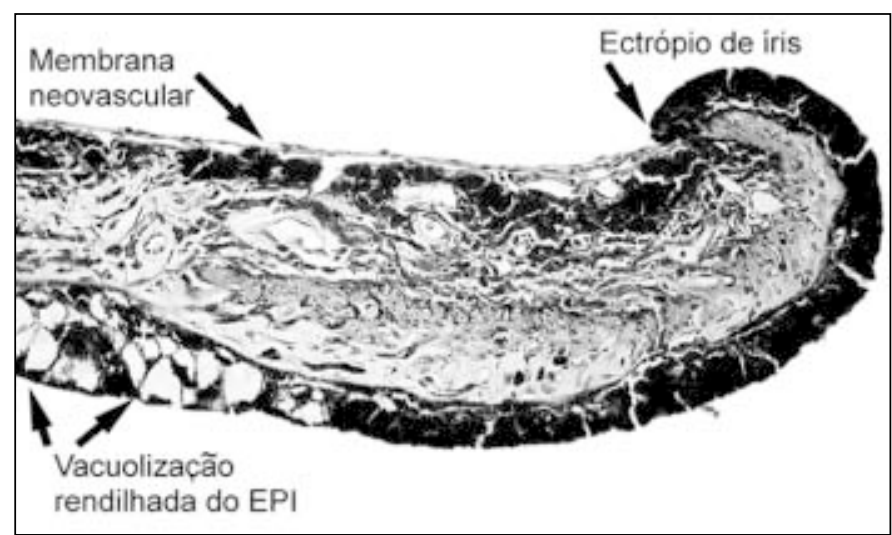

Figura 4 - Corte histológico da íris com neovascularização (20X). Observe a membrana fibro-vascular que se adere à superfície da íris causando tração e ectrópio de íris bem como as alterações do epitélio pigmentado da íris

diabéticos, o que sugere sua relação com altos níveis de glicose sérica. A glicogênese focal do epitélio pigmentar da íris deve ser análoga ao acúmulo de glicogênio no túbulos renais conhecida como nefropatia glicogênica de ArmanniEbstein $^{(24)}$. O dano tecidual causado pela vacuolização rendilhada deve ser responsável pela dispersão pigmentar do humor aquoso durante o intra-operatório de alguns olhos diabéticos. Essa vacuolização rendilhada pode ser evidenciada clinicamente como um padrão "roído de traça" na transiluminação ou biomicroscopia da íris ${ }^{(24)}$.

Um espessamento da membrana basal ocorre de forma sistêmica no diabetes mellitus conforme explicado anteriormente sobre a retina. Esse espessamento, na camada pigmentada interna do epitélio pigmentar da íris, permite distinguir histopatologicamente se um olho com glaucoma neovascular tem diabetes ${ }^{(15)}$. $\mathrm{O}$ espessamento da membrana basal do epitélio corneano pode predispor a uma descamação desse epitélio durante cirurgia vítreo-retiniana ${ }^{(7)}$.

\section{ABSTRACT}

Diabetic retinopathy continues to be an important cause of adult blindness among Americans and Brazilians. There are determinant environmental factors in the development of diabetic retinopathy although increasing evidence suggests a genetic component in diabetic retinopathy. The advances made during the last two decades with the purpose of improving the understanding of the mechanisms involved in the development and progression of diabetic retinopathy have prompted this review. This article has the purpose of revising and updating concepts about the ocular physiopathology of diabetes mellitus.
Keywords: Diabetes mellitus, Diabetic retinopathy/physiopathology; Diabetic retinopathy/pathology; Retinal neovascularization

\section{REFERÊNCIAS}

1. The effect of intensive treatment of diabetes on the development and progres sion of long-term complications in insulin-dependent diabetes mellitus. The Diabetes Control and Complications Trial Research Group. N Engl J Med. 1993;329(14):977-86. Comment in: ACP J Club. 1994;120(Suppl 2):30-1.

2. Olk RJ, Lee CM. Diabetic retinopathy: practical management., 1st ed. Philadelphia: Lippincott Company, 1993;39.

3. Dorchy H. Characterization of early stages of diabetic retinopathy. Diabetes Care. 1993;16(8):1212-4. Comment on: Diabetes Care.15(12):1844-74.

4. Khadem JJ, Buzney SM, Alich KS. Practice patterns in diabetic retinopathy: part 1: analysis of retinopathy follow-up. Arch Ophthalmol. 1999;117(6):815-20.

5. Corrêa ZMS, Freitas AM, Marcon IM. Risk factors related to the severity of diabetic retinopathy. Arq Bras Oftalmol. 2003;66(6):739-43.

6. Warpeha KM, Chakravarthy U. Molecular genetics of vascular disease in diabetic retinopathy. Eye. 2003;17(Pt 3):305-11.

7. Eagle RC Jr. The ocular pathology of diabetes mellitus. In: Rapuano CJ, editor. Yearbook of Ophthalmology. Philadelphia: Mosby, 1994. p.357-64.

8. Scheffel RS, Bortolanza D, Weber CS, Costa LA da, Canani LH, Santos KG, et al. Prevalência de complicações micro e macrovasculares e de seus fatores de risco em pacientes com diabetes melito do tipo 2 em atendimento ambulatorial. Rev Assoc Med Bras. 2004;50(3):263-7.

9. Freitas AM, Corrêa ZMS, Marcon IM, Schmidt H. A proteinúria como fator de risco para o desenvolvimento da retinopatia diabética. Arq Bras Oftalmol. 2002;65(1):83-7.

10. Pereira DS, Reis FAC, Waetge RTL, Caliari LE, Aihara T. Prevalência da retinopatia diabética no Ambulatório de Endocrinologia Pediátrica da Santa Casa de Misericórdia de São Paulo. Arq Bras Oftalmol. 2004;67(1):111-4.

11. Gross JL, Nehme M. Detecção e tratamento das complicações crônicas do diabetes melito: Consenso da Sociedade Brasileira de Diabetes e Conselho Brasileiro de Oftalmologia. Rev Assoc Med Brasil. 1999;45(3):279-84.

12. Schellini SA, Silva MRBM, Silva MAM. Diabetes, retinopatia diabética e cegueira. J Bras Med. 1994;67(2):171-4.

13. Frank RN. Etiologic mechanisms in diabetic retinopathy. In: Ryan SJ, Retina. 2nd ed. St Louis: Mosby, 1994. Vol.2. p.1243-76.

14. Effect of intensive therapy on the development and progression of diabetic nephropathy in the Diabetes Control and Complications Trial. Kidney Int. 1995;47(6):1703-20.

15. Garner A. Histopathology of diabetic retinopathy in man. Eye. 2003;7(Pt 2): 250-3.

16. Cunha Vaz JG, Fonseca JR, Abreu JF, Ruas MA. Detection of early retinal changes in diabetes by vitreous fluorophotometry. Diabetes. 1979;28(1):16-9.

17. Wolter JR. Pathology of a cotton wool-spot. Am J Ophthalmol. 1959;48:473-85.

18. Cogan DG, Kuwabara T. Comparison of retinal and cerebral vasculature in trypsin digest preparations. Br J Ophthalmol. 1984;68(1):10-2.

19. Joussen A, Poulaki V, Le ML, Koizumi K, Esser C, Janicki H, et al. A central role for inflammation in the pathogenesis of diabetic retinopathy. FASEB. 2004;18(12):1450-2.

20. Stanford MR. The pathogenesis of diabetic retinopathy. Br J Ophthalmol. 2004;88(4):444-5.

21. Aiello LP, Cahill MT, Cavallerano JD. Growth factors and protein kinase C inhibitors as novel therapies for the medical management of diabetic retinopathy. Eye. 2004;18(2):117-25.

22. Hughes JM, Brink A, Witmer AN, Witmer AN, Hanraads-de Riemer M, Klaassen I, et al. Vascular leucocyte adhesion molecules unaltered in the human retina in diabetes. Br J Ophthalmol. 2004;88(4):566-72.

23. John T, Sassani JW, Eagle RC Jr. The myofibroblastic component of rubeosis iridis. Ophthalmology. 1983;90(6):721-8.

24. Smith ME, Glickman P. Diabetic vacuolization of the iris pigment epithelium. Am J Ophthalmol. 1975;79(5):875-7. 